OPEN ACCESS

Edited by:

Salah D. Qanadli,

University of Lausanne, Switzerland

Reviewed by:

Gilles Soulez,

University of Montreal Hospital Centre

(CRCHUM), Canada

Kan Zen,

Kyoto Prefectural University

of Medicine, Japan

${ }^{*}$ Correspondence:

Min Yang

dryangmin@gmail.com

Jianxing Qiu

qjx761225@126.com

tThese authors have contributed equally to this work and share first

authorship

Specialty section:

This article was submitted to

Cardiovascular Imaging,

a section of the journal

Frontiers in Cardiovascular Medicine

Received: 12 October 2021

Accepted: 07 February 2022

Published: 28 February 2022

Citation:

Lin Z, Zhang B, Lin L, Wang $R$,

Niu G, Yan Z, Zou Y, Tong X, Qiu J and Yang $M$ (2022) Prediction of Split

Renal Function Improvement After

Renal Artery Stenting by Blood Oxygen Level-Dependent Magnetic

Resonance Imaging.

Front. Cardiovasc. Med. 9:793777.

doi: 10.3389/fcvm.2022.793777

\section{Prediction of Split Renal Function Improvement After Renal Artery Stenting by Blood Oxygen Level-Dependent Magnetic Resonance Imaging}

\author{
Zhiyong Lin ${ }^{1,2+}$, Bihui Zhang ${ }^{1+}$, Letao Lin 1,3,4t, Rui Wang ${ }^{2}$, Guochen Niu', Ziguang Yan', \\ Yinghua Zou ${ }^{1}$, Xiaoqiang Tong ${ }^{1}$, Jianxing Qiu ${ }^{2 *}$ and Min Yang ${ }^{1 *}$
}

\begin{abstract}
${ }^{1}$ Department of Interventional Radiology and Vascular Surgery, Peking University First Hospital, Beijing, China, ${ }^{2}$ Department of Radiology, Peking University First Hospital, Beijing, China, ${ }^{3}$ State Key Laboratory of Oncology in South China, Collaborative Innovation Center for Cancer Medicine, Sun Yat-sen University Cancer Center, Guangzhou, China, ${ }^{4}$ Minimally Invasive Interventional Division, Sun Yat-sen University Cancer Center, Guangzhou, China
\end{abstract}

Background: The discrepancy between the high technical success rate and the relatively low clinical response rate of renal artery stenting (RAS) raises the importance to identify atherosclerotic renal artery stenosis (ARAS) patients who are most likely to benefit from RAS. This study aimed to investigate the feasibility and accuracy of blood oxygen level-dependent magnetic resonance imaging (BOLD-MRI) in predicting split renal function (SRF) improvement after RAS in patients with ARAS.

Methods: Thirty patients with severe ARAS who were treated with RAS were enrolled. Baseline cortical and medullary R2* values of each kidney were measured by BOLD$\mathrm{MRI}$, and each patient's SRF was evaluated by nuclear renal dynamic imaging at baseline and 1-month follow-up.

Results: In total, 35 severe stenotic renal arteries of the 30 patients were analyzed. At 1-month follow-up, 34 kidneys (97.1\%) of severe ARAS had acquired SRF. SRF improved in 12 kidneys of 10 patients. The cortical R2* and medullary R2* values in the SRF improvement kidneys were higher than those in the non-improvement kidneys $(P \leq 0.001)$. The area under the curve of medullary R2* was 0.879 (95\% confidence interval $[\mathrm{Cl}]$ 0.736-1.000). A medullary $\mathrm{R} 2 *$ value $\geq 29.1 \mathrm{~s}^{-1}$ was noted to provide good sensitivity $(0.833,95 \% \mathrm{Cl} 0.552-0.970)$ and specificity $(0.864,95 \% \mathrm{Cl} 0.667-0.953)$ in predicting SRF improvement. Medullary R2* value was the only independent predictor of SRF improvement in multivariable analysis $(P=0.034$, OR 3.017, 95\% Cl 1.089-8.358).

Conclusion: This study showed that a BOLD-MRI medullary R2* value $\geq 29.1 \mathrm{~s}^{-1}$ was an excellent predictor of SRF improvement in patients with severe ARAS who underwent renal artery stenting.

Keywords: atherosclerotic renal artery stenosis, renovascular stenting, renal artery stenting, blood oxygen leveldependent magnetic resonance imaging, prediction 


\section{INTRODUCTION}

Atherosclerotic renal artery stenosis (ARAS) is the most common cause of secondary hypertension, with severe stenosis $(>60 \%)$ occurring in $6.8 \%$ of elderly patients (1-3). Renal artery stenting (RAS) is the preferred treatment option. Still, recent randomized trials have reported that when RAS is combined with the best medical therapy, there is no benefit regarding blood pressure, renal function, cardiovascular events, or mortality compared to medical treatment alone (4-6). However, these trials had significant design flaws, including variability in inclusion and exclusion criteria, inconsistent definitions of improvement, and differing endpoints, making the selection of patients for renal artery stenting controversial (7-9). For this reason, determining a biomarker to help predict RAS outcomes and identify patients who would best respond to RAS is of great clinical significance $(8,9)$.

Currently, several biomarkers are used to select patients likely to benefit from RAS, including resistance index (RI), translesional pressure gradient, renal artery fractional flow reserve, and brain natriuretic peptide (10). However, these methods are either invasive or not sufficiently accurate to be applied widely. Blood oxygen level-dependent magnetic resonance imaging (BOLD$\mathrm{MRI}$ ) is a non-invasive imaging technique that can assess the kidney's regional oxygenation (11). It uses endogenous deoxygenated hemoglobin as a contrast agent and detects magnetic field disturbances caused by changes of oxygenated hemoglobin within the kidney tissue (11). The $\mathrm{R} 2 *$ score is the magnetic rate of relaxation that correlates positively with deoxyhemoglobin levels and can evaluate the severity of ischemia caused by ARAS $(12,13)$.

The present study evaluated the feasibility and accuracy of using BOLD-MRI to identify severe ARAS patients who might benefit from RAS in split renal function (SRF).

\section{MATERIALS AND METHODS}

\section{Patients}

This prospective study enrolled patients with severe ARAS consecutively from May 2018 to November 2018 and was approved by the Human Investigations Committee of Peking University First Hospital (2018263). The inclusion criteria were as follows: (1) patient age was $\geq 40$ years; (2) the degree of stenosis on the treatment side of the renal artery was $\geq 70 \%$ (including occlusion), and there was no definite stenosis in the branches of the renal artery; (3) Renal artery stenosis was caused by atherosclerosis, which included (1) a minimum of 1 atherosclerosis risk factor (age $\geq 40$, diabetes, hyperlipidemia, obesity, or long-term smoking) and (2) a minimum of 2 imaging features of atherosclerosis (the lesion involved the ostium or proximal segment of the renal artery, eccentric stenosis or occlusion, irregular plaque, calcification, and signs of atherosclerosis in other abdominal vessels); (4) clinical symptoms related to renal artery stenosis were present, including hypertension (blood pressure $>140 / 90 \mathrm{mmHg}$ ), impaired renal function (taking renal dynamic imaging as the reference standard, unilateral glomerular filtration rate (GFR) $<34 \mathrm{ml} / \mathrm{min}$ ), or acute pulmonary edema and unstable angina pectoris; and (5) patients signed informed consent. Exclusion criteria mainly included the following: Renal artery stenosis caused by non-atherosclerotic factors, such as Takayasu arteritis, fibromuscular dysplasia, congenital arterial malformation, restenosis after renal artery intervention, or graft RAS; severe renal dysfunction [serum creatinine $>264 \mu \mathrm{mol} / \mathrm{L}$ $(3.0 \mathrm{mg} / \mathrm{dl})]$; and atrophied kidney (length $<7 \mathrm{~cm}$ ). The complete inclusion and exclusion criteria are shown in the appendix. SRF was evaluated with radionuclide renal dynamic imaging and the GFRs of both kidneys were obtained at baseline and 1-month follow-up.

\section{Endovascular Procedures}

Patients were treated by experienced vascular specialists in a catheter lab. Local anesthesia was administered and supplemented with intravenous sedation when needed. At first, a renal artery digital subtraction angiography was performed, and the operator determined the degree of stenosis based on the angiography images. In terms of diameter stenosis, less than $50 \%$ was considered mild, $50-70 \%$ moderate, $70-99 \%$ severe, and $100 \%$ occlusion. For the patients with severe stenoses and occlusions, endovascular treatments were performed. A 7F short sheath was exchanged for artery access, a 7F guiding catheter was used to engage the ostium of the stenotic renal artery, and a 0.014inch guidewire was manipulated to cross the stenotic lesion. A rapid exchange balloon, 3-5 $\mathrm{mm}$ in diameter, was advanced along the guidewire to predilate the lesion. After predilation, a balloon-expandable stent was induced through the guiding catheter and deployed at the stenotic segment. The length and diameter of the balloons and stents were chosen at the discretion of the operator. Completion angiography was performed to confirm procedural success. Technical success was defined as a $<30 \%$ residual stenosis at the completion angiography.

\section{Antiplatelet and Antihypertensive Protocol}

Before the procedure, all patients underwent dual antiplatelet therapy (aspirin $100 \mathrm{mg} /$ day and clopidogrel $75 \mathrm{mg} /$ day) for at least 7 days or took a loading dose (aspirin $300 \mathrm{mg}$ and clopidogrel $300 \mathrm{mg}) 6 \mathrm{~h}$ before the procedure. During the procedure, heparin (3000 IU) was administered for anticoagulation after the insertion of a guiding catheter. All patients were prescribed dual antiplatelet therapy (aspirin $100 \mathrm{mg} /$ day and clopidogrel $75 \mathrm{mg} /$ day) for at least 3 months after the procedure and were converted to a single agent after that. Statin was prescribed for patients with dyslipidemia. Antihypertensive drugs were kept unchanged at baseline and after the endovascular treatment.

\section{Magnetic Resonance Imaging Scan Protocol}

All BOLD-MRI examinations were performed with a MAGNETOM Aera 1.5T MR scanner (Siemens Healthcare $\mathrm{GmbH}$, Erlangen, Germany) using an 18-channel body 
coil combined with a 32-channel spine coil. The BOLDMRI examination was a plain scan without the use of contrast agents. A coronal position protocol of multipleecho spoiled gradient recalled echo through the center of both kidneys was prescribed. The parameters were as follows: repetition time, $130 \mathrm{msec} 5$ echoes (echo times: 9.43, 14.29, 19.05, 23.81, and $28.30 \mathrm{msec}$ ); flip angle, $90^{\circ}$; slices, 4; section thickness, $6 \mathrm{~mm}$; slice gap, $1.8 \mathrm{~mm}$; imaging matrix, $256 \times 192$; and field of view, $380 \mathrm{~mm} \times 297 \mathrm{~mm}$. The scanning sequence automatically calculated and produced the $\mathrm{T} 2 *$-weighted mapping images.

\section{Magnetic Resonance Imaging Quality Evaluation and Data Analysis}

Two radiologists independently assessed the image quality of the BOLD-MRI in terms of image clarity, artifacts, and geometric distortion. Image quality was rated according to a 5-point Likerttype scale (1, non-diagnostic; 2 , poor; 3 , moderate; 4 , good; and 5, excellent).

Analysis of BOLD-MRI data from coronal position images was performed by drawing a parenchymal region of interest (ROI) on the sections through each kidney's mid pole hilar region on the T2*-weighted mapping images. Four ROIs were traced: One ROI of cortex was calculated after manual segmentation of the cortex while paying attention excluding the renal medulla, collecting system, incidental cysts, and hilar structures; three ROIs of the medulla were placed in circular areas on the upper, middle, and lower medulla of the kidney, with a diameter of about $3 \mathrm{~mm}$ (Figure 1).
After being trained in the standard unified measurement process, two radiologists measured the $\mathrm{T} 2 *$ values of the BOLDMRI images separately in a blinded fashion. Any discrepancies were resolved in a consensus reading. The final T2* values were the average of the data measured by the two radiologists.

One T2* value was obtained for the cortex of each kidney. Three $\mathrm{T} 2 *$ values of ROIs were obtained for the medulla, and the average was calculated. Finally, to evaluate the degree of hypoxia in the kidney tissue, these values were converted into R2* values according to the following formula: $\mathrm{R} 2 *=1 / \mathrm{T} 2 *$.

\section{Follow-Up and Definitions}

Follow-up visits were scheduled at 1-month after discharge at the outpatient department. During the follow-up appointment, symptom inquiry, physical examination, renal artery duplex ultrasound scanning and nuclear renal dynamic imaging were conducted. The renal artery ultrasound measurements included peak systolic velocity (PSV) and RI. In the nuclear renal dynamic imaging scan, a bolus of 99mTc-DTPA (Technetium99m diethylene triamine penta-acetic acid) was given through an antecubital vein. For renal blood flow evaluation, serial dynamic images are acquired every $2 \mathrm{~s}$ for a minute. To evaluate renal parenchymal function, a series of images are obtained after $1 \mathrm{~min}$ of the renal perfusion phase. Images are acquired at $60 \mathrm{~s}$ intervals for a period of $20 \mathrm{~min}$.

Loss of patency was defined as $\geq 50 \%$ restenosis or stent thrombosis based on duplex ultrasound scanning. Change of GFR was defined as the difference between the GFR taken at the 1-month follow-up and the baseline. A modified definition of SRF

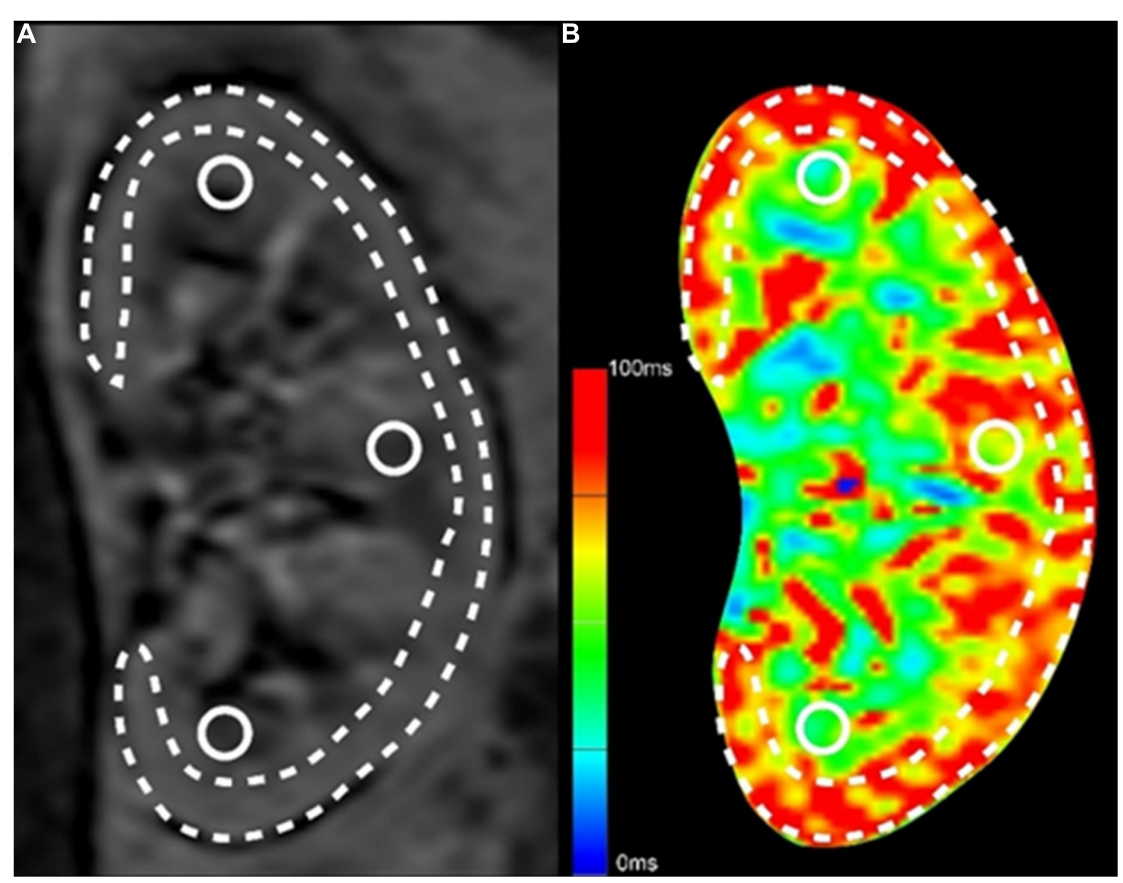

FIGURE 1 | Methods for ROI selection on BOLD-MRI images. (A) The original T2*-weighted image; (B) The pseudo-color T2*-weighted image. The long and narrow area depicted by the dashed line was the ROI of renal cortex, excluding the renal medulla, collecting system, incidental cysts, and hilar vessels. The three circular areas on the upper, middle and lower medulla of the kidney were the ROls of the medulla. 
improvement was the change of GFR was $\geq 15 \%$ of the baseline GFR of the affected kidney and the absolute improvement was at least $5 \mathrm{ml} / \mathrm{min}$.

\section{Statistical Analysis}

Statistical analysis was performed using SPSS software (version 20.0, IBM Corp., Armonk, NY, United States). The normally distributed continuous data are shown as mean \pm standard deviation, continuous data without normal distribution are shown as the median and interquartile, and categorical data are shown as numbers and percentages. The normally distributed paired continuous variables were compared by 2-sided Student's $t$-tests, and the non-normally distributed paired continuous variables were compared by the Wilcoxon signed-rank test. Mean values of independent variables were compared using an independent samples $t$-test when the homogeneity of variance was present and using Welch's $t$-test when the variance homogeneity was absent. Categorical variables were compared using the 2-sided likelihood ratio chi-square test or Fisher exact test.

Pearson correlation coefficient was used to evaluate the correlation between the two groups of data with normal distribution, and Spearman correlation coefficient was used to evaluate the correlation between the two groups of data without normal distribution. Furthermore, $0.4 \leq|\mathrm{r}|<0.7$ was considered a moderate correlation, $0.7 \leq|\mathrm{r}|<0.9$ a high correlation, and $|\mathrm{r}|$ $\geq 0.9$ a very high correlation.

The kappa value was used to analyze image quality consistency through a grade score, and the intraclass correlation coefficient (ICC) was used to evaluate the consistency of data measurement. The consistency was reported as poor when ICC $<0.4$, good when $0.4 \leq$ ICC $\leq 0.75$, and very good when ICC $>0.75$.

Receiver operating characteristic (ROC) curve analysis was performed to evaluate baseline cortical and medullary R2* performance across the patients analyzed. The area under the curve (AUC) was used to assess the overall accuracy. Youden's index and clinical relevance would be considered when selecting the optimum cut-off point. Univariate and multivariate logistic regression were used to analyze the risk factors of the improvement of SRF after RAS. Characteristics of patients and lesions, and BOLD parameters were placed in the analysis model.

Outcomes were reported as the hazard ratio and $95 \%$ confidence interval (CI). A $P$-value of less than 0.005 was considered statistically significant.

\section{RESULTS}

\section{Patient and Procedural Characteristics}

A total of 30 patients with ARAS were included in this study. The flowchart of patients is shown in Figure 2. Demographic and baseline information is shown in Table 1. The average age was $63.97 \pm 9.44$ years, and $73.3 \%$ of patients were male. All patients suffered from high blood pressure, and the median number of antihypertensive agents was 2 .

Bilateral renal artery lesions were found in 9 patients, 4 of whom had unilateral renal artery occlusion that could

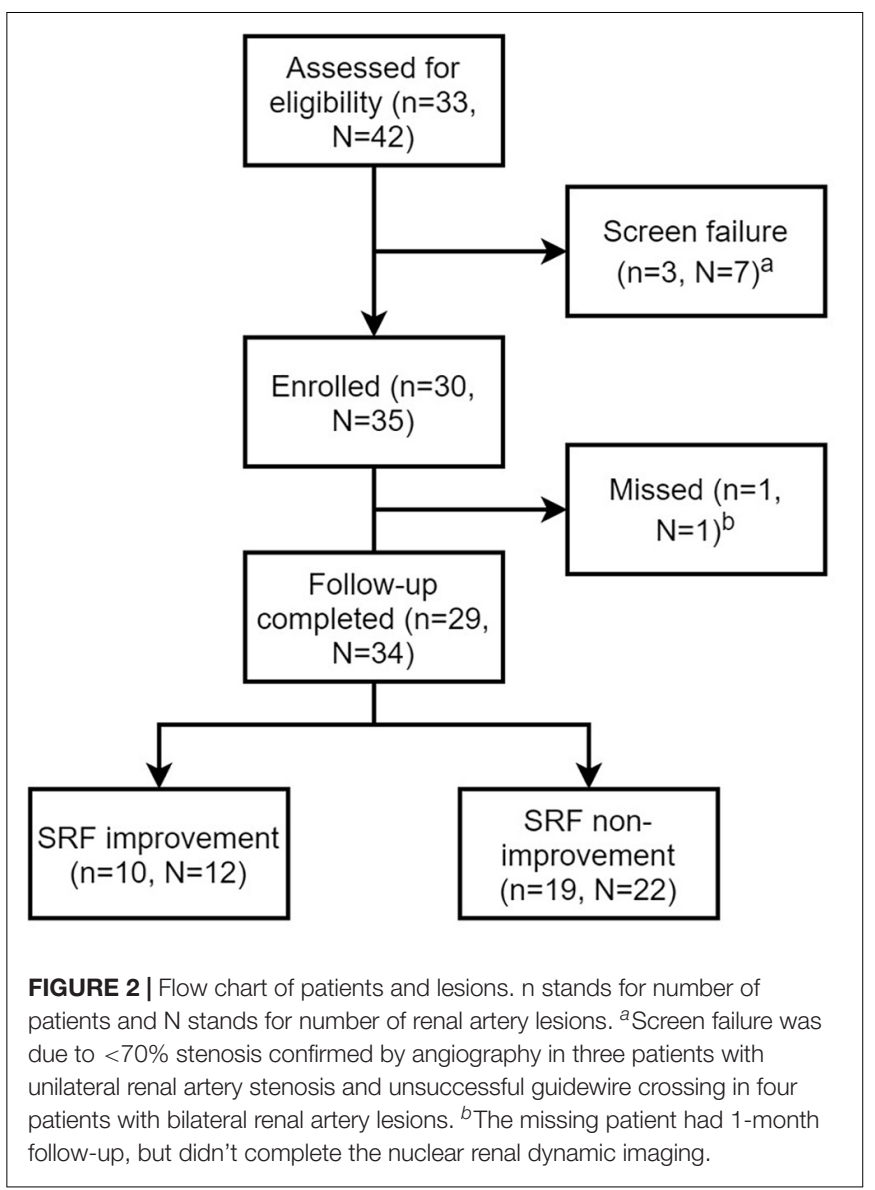

TABLE 1 | Patient characteristics $(n=30)$.

\begin{tabular}{|c|c|}
\hline Characteristics & $N=30$ \\
\hline Age, years & $63.97 \pm 9.44$ \\
\hline Male, $n(\%)$ & $22(73.30)$ \\
\hline $\mathrm{BMI}\left(\mathrm{kg} / \mathrm{m}^{2}\right)$ & $25.00 \pm 2.71$ \\
\hline Diabetes, $n(\%)$ & $8(26.70)$ \\
\hline Dyslipidemia, $n(\%)$ & $17(56.70)$ \\
\hline Cerebrovascular disease, $n(\%)$ & $6(20.00)$ \\
\hline Coronary heart disease, $n(\%)$ & $12(40.00)$ \\
\hline Peripheral artery disease, $n(\%)$ & $2(6.70)$ \\
\hline Smoking, $n(\%)$ & $14(46.7)$ \\
\hline Hypertension, $n$ (\%) & $30(100.00)$ \\
\hline Duration of hypertension, years & $4(1.00,10.00)$ \\
\hline Systolic blood pressure (mmHg) & $160.77 \pm 20.20$ \\
\hline Diastolic blood pressure $(\mathrm{mmHg})$ & $87.70 \pm 11.56$ \\
\hline Kinds of antihypertensive agents & $2(1.00,3.00)$ \\
\hline Use of ACEI/ARB, $n(\%)$ & $12(40.00)$ \\
\hline Use of diuretics, $n(\%)$ & $7(23.30)$ \\
\hline Use of $\beta$-Blockers & $12(40.00)$ \\
\hline Renal insufficiency, $n$ (\%) & $11(36.67)$ \\
\hline Serum creatinine $(\mu \mathrm{mol} / \mathrm{L})$ & $125.75(97.06,147.94)$ \\
\hline eGFR (mL/min/1.73m²) & $52.64 \pm 18.72$ \\
\hline
\end{tabular}

BMI, Body Mass Index; ACEl, Angiotensin Converting-Enzyme Inhibitor; ARB, Angiotensin II Receptor Blocker; eGFR, estimated Glomerular Filtration Rate; Renal insufficiency was defined as serum creatinine $>133 \mu \mathrm{mol} / \mathrm{L}$. 
not be recanalized successfully, and unilateral ARAS was found in 21 patients who all undertook RAS successfully. Overall, 35 renal arteries were treated by stents (31 PALMAZ BLUE, Cordis, FL, United States; 1 Herculink Elite, Abbott Vascular, CA, United States;1 Acculink, Abbott Vascular, CA, United States and 2 Express Vascular, Boston Scientific, MA, United States).

\section{Quality and Consistency of Blood Oxygen Level-Dependent Imaging}

The quality of the acquired images met the need for diagnosis regarding resolution, artifacts, and deformation. The kappa value of the image quality was 0.816 .

A total of 35 kidneys with severe ARAS were included for evaluation of cortical and medullary $\mathrm{T}^{*}$ at baseline. The ICC was 0.921 (95\% CI 0.887-0.945) for cortical $\mathrm{T}^{*}$ and 0.818 (95\% CI $\left.0.74-0.871\right)$ for medullary $\mathrm{T} 2 *$, indicating that the two radiologists noted a high consistency in estimating both cortical and medullary $\mathrm{T} 2 *$.

\section{Oxygenation Level of Kidneys With Severe Atherosclerotic Renal Artery Stenosis}

The oxygenation level of affected kidneys was compared with the contralateral kidneys in the 21 patients with severe unilateral ARAS. The cortical and medullary T2* values of the affected kidneys were significantly lower than those of the contralateral kidneys ( $P=0.007$ and $P<0.001$, respectively). Accordingly, the R2* $\left(1 / \mathrm{T} 2^{*}\right)$ values were higher in the affected side than in the

TABLE 2 | BOLD-MRI comparison of the affected kidney and contralateral kidney in patients with unilateral ARAS.

\begin{tabular}{llccr}
\hline & & $\begin{array}{c}\text { The severely stenotic } \\
\text { kidney }(\boldsymbol{n}=\mathbf{2 1})\end{array}$ & $\begin{array}{c}\text { The contralateral } \\
\text { kidney }(\boldsymbol{n}=\mathbf{2 1})\end{array}$ & $\boldsymbol{P}$ \\
\hline Cortical & $\mathrm{T} 2{ }^{*}(\mathrm{~ms})$ & $78.12 \pm 10.86$ & $85.16 \pm 5.50$ & 0.007 \\
& $\mathrm{R} 2^{*}\left(\mathrm{~s}^{-1}\right)$ & $13.03 \pm 1.78$ & $11.79 \pm 0.74$ & 0.003 \\
\multirow{3}{*}{ Medullary } & $\mathrm{T}^{*}(\mathrm{~ms})$ & $38.37 \pm 4.96$ & $44.16 \pm 2.59$ & $<0.001$ \\
& $\mathrm{R} 2^{*}\left(\mathrm{~s}^{-1}\right)$ & $26.48 \pm 3.38$ & $22.72 \pm 1.35$ & $<0.001$ \\
\hline
\end{tabular}

BOLD-MRI, Blood Oxygen Level-Dependent Magnetic Resonance Imaging; ARAS, Atherosclerotic Renal Artery Stenosis. contralateral side $(P=0.003$ and $P<0.001$, respectively). Details are shown in Table 2.

\section{One-Month Outcomes of Endovascular Treatment}

All patients with severe ARAS had a 1-month followup. The parameters of global renal function and blood pressure at both the baseline and follow-up are given in Table 3. The average systolic blood pressure decreased from $160.77 \pm 20.20 \mathrm{mmHg}$ at baseline to $132.80 \pm 10.56 \mathrm{mmHg}$ at the 1 -month follow-up $(P<0.001)$. The average diastolic blood pressure decreased from $87.70 \pm 11.56 \mathrm{mmHg}$ to $78.27 \pm 9.00 \mathrm{mmHg}(P<0.001)$. Parameters regarding global renal function did not change.

For the 35 renal arteries treated and evaluated at followup, no loss of patency was found by ultrasound (Table 4). The velocity of renal artery flow decreased significantly $(0.98 \pm 0.34 \mathrm{~m} / \mathrm{s}$ vs. $2.96 \pm 1.45 \mathrm{~m} / \mathrm{s}, P<0.001)$. One patient did not complete the nuclear renal dynamic imaging at the 1-month follow-up. SRF improvement occurred in $35.3 \%(12 / 34)$ of the stented kidneys in $34.5 \%$ $(10 / 29)$ of the patients. The average split GFR increased significantly $(19.56 \pm 11.86 \mathrm{ml} / \mathrm{min}$ vs. $17.16 \pm 11.69 \mathrm{ml} / \mathrm{min}$, $P=0.013)$.

\section{The Correlation of Oxygenation Level at Baseline and Change of Glomerular Filtration Rate}

Correlations between the baseline oxygenation level of the renal cortex and medulla, and the 1-month change of ipsilateral GFR were analyzed in the 34 kidneys treated by RAS. Pearson correlation analysis showed that there was a moderate positive correlation between the GFR change and baseline cortical R2* value $(r=0.674 ; P<0.001$; Figure 3A)

TABLE 4 | The nuclear renal dynamic and Doppler ultrasound parameters of stented kidneys at baseline and 1-month follow-up $(n=35)$.

\begin{tabular}{lccc}
\hline Parameters & Baseline & 1 month & P-value \\
\hline GFR (ml/min) & $17.16 \pm 11.69$ & $19.56 \pm 11.86$ & 0.013 \\
PSV (m/s) & $2.96 \pm 1.45$ & $0.98 \pm 0.34$ & $<0.001$ \\
RI & $0.69 \pm 0.13$ & $0.71 \pm 0.09$ & 0.298
\end{tabular}

GFR, Glomerular Filtration Rate; PSV, Peak Systolic Velocity; RI, Resistance Index.

TABLE 3 | Comparison of global renal function and blood pressure at baseline and 1-month follow-up.

\begin{tabular}{|c|c|c|c|}
\hline Variables & At baseline $(n=30)$ & At 1-month follow up $(n=30)$ & $P$ \\
\hline Serum creatinine $(\mu \mathrm{mol} / \mathrm{L})$ & $125.75(97.06,147.94)$ & $118.50(97.43,146.25)$ & 0.951 \\
\hline eGFR $\left(\mathrm{mL} / \mathrm{min} / 1.73 \mathrm{~m}^{2}\right)$ & $52.64 \pm 18.72$ & $53.39 \pm 17.16$ & 0.695 \\
\hline Urine creatinine (mmol/L) & $4.70(3.85,7.45)$ & $7.05(4.78,12.40)$ & 0.063 \\
\hline Urine microalbumin (mg/L) & $27.90(3.91,166.50)$ & $22.35(6.23,63.70)$ & 0.370 \\
\hline Systolic blood pressure (mmHg) & $160.77 \pm 20.20$ & $132.80 \pm 10.56$ & $<0.001$ \\
\hline Diastolic blood pressure (mmHg) & $87.70 \pm 11.56$ & $78.27 \pm 9.00$ & $<0.001$ \\
\hline
\end{tabular}

eGFR, estimated Glomerular Filtration Rate. 

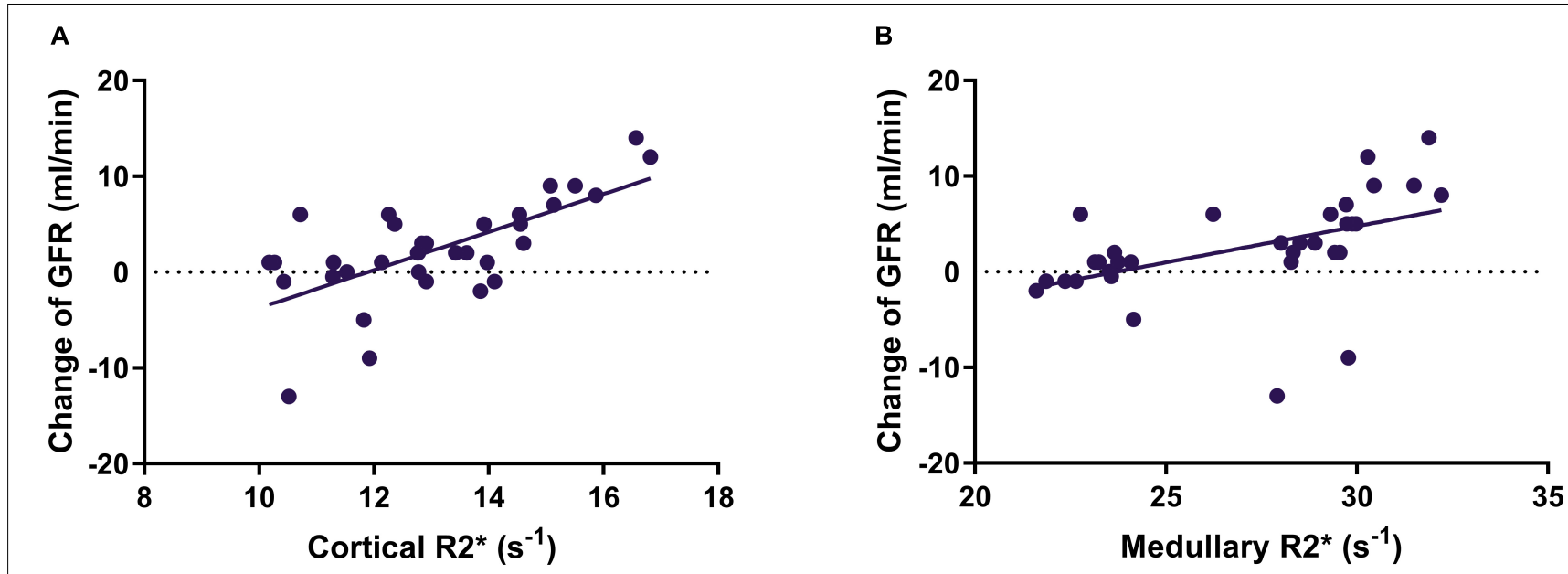

FIGURE 3 | The correlation between cortical R2* (A) and medullary R2* (B) and change of SRF of the stented kidney. SRF, split renal function; GFR, glomerular filtration rate.
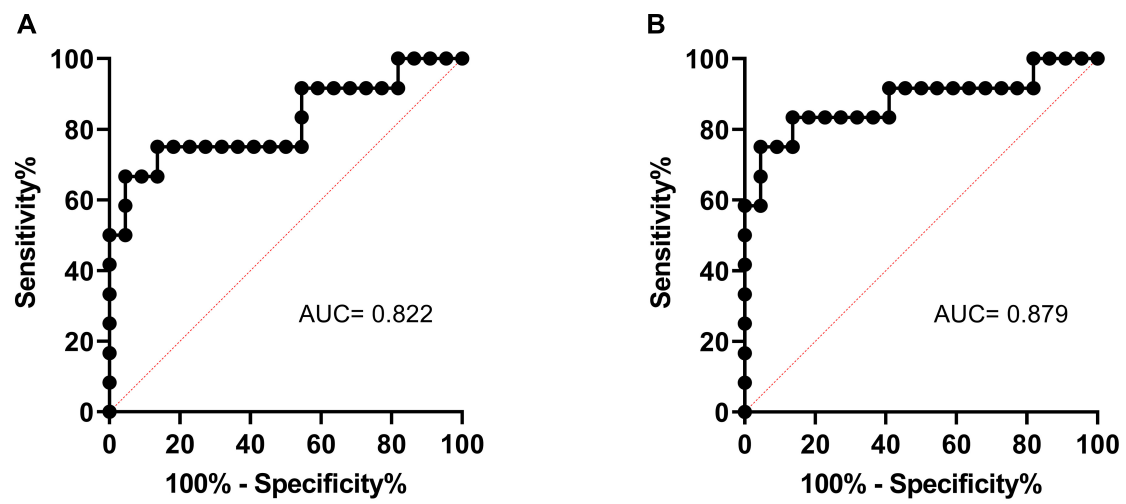

FIGURE 4 | The ROC curve of cortical R2* (A) and medullary R2* (B) in predicting SRF improvement. ROC, receiver operating characteristic; SRF, split renal function.

and baseline medullary $\mathrm{R}^{*}$ value $(r=0.483 ; P=0.004$; Figure 3B).

The baseline cortical $\mathrm{R} 2 *$ and medullary $\mathrm{R} 2 *$ in the SRF improvement group $\left(14.4 \pm 1.8 \mathrm{~s}^{-1}\right.$ and $29.5 \pm 2.6 \mathrm{~s}^{-1}$, respectively) were higher $(P=0.001$ and $P<0.001$, respectively) than those in the non-improvement group (12.4 $\pm 1.3 \mathrm{~s}^{-1}$ and $25.4 \pm 2.9 \mathrm{~s}^{-1}$, respectively), indicating that baseline cortical $\mathrm{R} 2 *$ and medullary $\mathrm{R} 2 *$ values could be candidates for predicting SRF improvement. For patients with SRF improvement, the serum creatinine level and eGFR did not change significantly $(P=0.321$ and $P=0.228)$.

\section{The Correlation of Oxygenation Level at Baseline and Change of Blood Pressure}

The correlations of BOLD-derived renal oxygenation level and evolution of blood pressure were analyzed in 25 patients treated by RAS unilaterally. The baseline medullary R2* had no correlation with absolute systolic blood pressure (SBP) change $(r=0.102$ and $P=0.628)$, relative SBP change $(r=0.138$ and $P=0.509)$, absolute diastolic blood pressure (DBP) change $(r=0.195$ and $P=0.352)$, and relative DBP change $(r=0.265$ and $P=0.200)$. Similarly, no correlation was found between the baseline cortical R2* and changes of blood pressure (absolute SBP $P=0.289$; relative SBP $P=0.308$; absolute $\mathrm{DBP} P=0.494$; relative DBP $P=0.484)$.

\section{The Role of R2* at Baseline in Predicting Split Renal Function Improvement}

The ROC curves of cortical R2* and medullary R2* are shown in Figure 4. The AUC of medullary R2* was 0.879 (95\% CI $0.736-1.000)$, larger than that of cortical R2* $(0.822,95 \%$ CI 0.655-0.989), indicating that medullary $\mathrm{R} 2 *$ had higher accuracy and is a better predictor. The cut-off value of baseline medullary R2* was $29.1 \mathrm{~s}^{-1}$, the sensitivity was $0.833(95 \%$ CI $0.552-0.970$ ), and the specificity was 0.864 (95\% CI $0.667-$ $0.953)$. The absolute values of GFR change were higher in patients with medullary $\mathrm{R} 2 *>29.1 \mathrm{~s}^{-1}$ than in patients with medullary R2* $\leq 29.1 \mathrm{~s}^{-1}(5.769 \pm 5.644$ vs. $0.309 \pm 3.945$, 
TABLE 5 | Univariable and Multivariable predictors of split renal function improvement after renal artery stenting.

\begin{tabular}{|c|c|c|c|c|c|c|c|c|}
\hline \multirow{3}{*}{$\begin{array}{l}\text { Predictors } \\
\text { Age }\end{array}$} & \multicolumn{4}{|c|}{ Univariable analysis } & \multicolumn{4}{|c|}{ Multivariable analysis } \\
\hline & \multirow{2}{*}{$\begin{array}{c}\boldsymbol{p} \text {-value } \\
0.462\end{array}$} & \multirow{2}{*}{$\frac{\text { OR }}{1.031}$} & \multicolumn{2}{|c|}{$95 \%$ confidence interval } & \multirow[t]{2}{*}{$p$-value } & \multirow[t]{2}{*}{ OR } & \multicolumn{2}{|c|}{$95 \%$ confidence interval } \\
\hline & & & 0.951 & 1.117 & & & & \\
\hline Male & 0.881 & 1.133 & 0.219 & 5.864 & & & & \\
\hline Smoking & 1.000 & 1.000 & 0.245 & 4.083 & & & & \\
\hline Duration of hypertension & 0.133 & 0.883 & 0.750 & 1.039 & & & & \\
\hline Diabetes & 0.346 & 2.333 & 0.400 & 13.609 & & & & \\
\hline Dyslipidemia & 0.611 & 1.444 & 0.351 & 5.947 & & & & \\
\hline Stroke & 0.881 & 0.882 & 0.171 & 4.565 & & & & \\
\hline Coronary artery disease & 0.966 & 0.969 & 0.232 & 4.042 & & & & \\
\hline Baseline GFR & 0.878 & 0.995 & 0.936 & 1.058 & & & & \\
\hline Degree of stenosis & 0.641 & 1.023 & 0.930 & 1.124 & & & & \\
\hline Baseline PSV & 0.217 & 1.402 & 0.820 & 2.396 & 0.549 & 0.798 & 0.381 & 1.671 \\
\hline Baseline RI & 0.510 & 0.163 & 0.001 & 36.047 & & & & \\
\hline Baseline kidney length & 0.353 & 1.352 & 0.715 & 2.554 & & & & \\
\hline Cortical R2* & 0.006 & 2.419 & 1.290 & 4.539 & & & & \\
\hline Medullary R2* & 0.006 & 1.674 & 1.157 & 2.422 & 0.034 & 3.017 & 1.089 & 8.358 \\
\hline
\end{tabular}

GFR, Glomerular Filtration Rate; PSV, Peak Systolic Velocity; RI, Resistance Index.

$P=0.002$ ). In the univariable model (Table 5), only cortical and medullary R2* were significantly associated with SRF improvement. Since cortical and medullary R2* were linearly correlated $(r=0.584, P<0.001)$ and PSV was considered clinically relevant, only medullary $\mathrm{R} 2 *$ and PSV were placed in the multivariable model. Medullary R2* was the only independent predictor of SRF improvement $(P=0.034$, OR 3.017, 95\%CI 1.089-8.358).

\section{DISCUSSION}

This pilot study elucidated that the R2* value of BOLD-MRI was an effective non-invasive biomarker for selecting ARAS patients who might benefit from RAS in SRF improvement. The AUC of medullary R2* was 0.879 (95\% CI $0.736-1.000)$, the sensitivity was 0.833 (95\% CI $0.552-0.970$ ), and the specificity was 0.864 (95\% CI 0.667-0.953) if the medullary R2* was $\geq 29.1 \mathrm{~s}^{-1}$.

Blood oxygen level-dependent magnetic resonance imaging is a non-invasive technique for evaluating kidney tissue oxygenation without requiring contrast exposure (14). A normally perfused kidney would be expected to have a low R2* score in the cortex and a higher R2* score in the deeper medullary region, consistent with the known hypoxia gradient in deep medullary areas (9). A low R2* score is observed if there is adequate perfusion and tissue function, but it can also be seen if the kidney is non-functioning, as the oxygenated blood passes through without oxygen utilization or consumption by atrophic tissue $(11,13)$. Therefore, high R2* appears when there is a significant decrease in renal blood flow and the kidney is still salvageable, which is exactly the condition in which ASAS patients may benefit from RAS. In the present study, the AUC of medullary R2* was 0.879 , indicating high accuracy in predicting SRF improvement after RAS.
The related randomized trials may have been confined by the use of global renal function as the outcome assessment (4-6). Due to the compensation achieved by hyperfiltering of the contralateral kidney in unilateral severe ARAS patients, there may be no net decline detected in the global renal function $(9,15,16)$. In the present study, nuclear dynamic imaging was used to assess the SRF improvement of the stented kidney. In the SRF improvement group, the global renal function evaluated by serum creatinine did not improve significantly, which supports the theory mentioned above (9). Blood pressure improvement is an important target for treating ARAS, and its predictors, including translesional pressure gradients, intravascular ultrasound, and angiography, have been previously investigated and compared (17). In the present study, the blood pressure improvement was not correlated with baseline cortical and medullary R2*. The possible mechanism is that blood pressure is associated with hemodynamic disturbance rather than the hypoxic condition.

The potential limitations of our study should be noted. First, SRF improvement was only evaluated at 1-month follow-up, and therefore long-term results remain unknown. The reason for the relatively short follow-up is that other comorbidities that ARAS patients have can adversely affect renal function. For this reason, it is difficult to determine whether RAS improves renal function in the long run. Second, the study was confined to a single center, and the bias associated with the sample size may limit the generalizability of the results. Third, the BOLD-derived oxygen levels cannot predict the outcomes of total GFR and blood pressure improvement, which limits the clinical impact.

\section{CONCLUSION}

Blood oxygen level-dependent magnetic resonance imaging is useful for identifying severe ARAS patients whose SRF could 
benefit from RAS. We found medullary R2* $\geq 29.1 \mathrm{~s}^{-1}$ to have good sensitivity and specificity, making it an excellent predictor of SRF improvement after RAS.

\section{DATA AVAILABILITY STATEMENT}

The raw data supporting the conclusions of this article will be made available by the authors, without undue reservation.

\section{ETHICS STATEMENT}

The studies involving human participants were reviewed and approved by Peking University First Hospital Ethics Committee. The patients/participants provided their written informed consent to participate in this study.

\section{REFERENCES}

1. Hansen KJ, Edwards MS, Craven TE, Cherr GS, Jackson SA, Appel RG, et al. Prevalence of renovascular disease in the elderly: a population-based study. $J$ Vasc Surg. (2002) 36:443-51. doi: 10.1067/mva.2002.127351

2. de Mast Q, Beutler JJ. The prevalence of atherosclerotic renal artery stenosis in risk groups: a systematic literature review. J Hypertens. (2009) 27:1333-40. doi: 10.1097/HJH.0b013e328329bbf4

3. Simeoni M, Borrelli S, Garofalo C, Fuiano G, Esposito C, Comi A, et al. Atherosclerotic-nephropathy: an updated narrative review. J Nephrol. (2021) 34:125-36. doi: 10.1007/s40620-020-00733-0

4. Bax L, Woittiez AJ, Kouwenberg HJ, Mali WP, Buskens E, Beek FJ, et al. Stent placement in patients with atherosclerotic renal artery stenosis and impaired renal function: a randomized trial. Ann Intern Med. (2009) 150:840-48; w150-841. doi: 10.7326/0003-4819-150-12-200906160-00119

5. Wheatley K, Ives N, Gray R, Kalra PA, Moss JG, Baigent C, et al. Revascularization versus medical therapy for renal-artery stenosis. $N$ Engl J Med. (2009) 361:1953-62. doi: 10.1056/NEJMoa0905368

6. Cooper CJ, Murphy TP, Cutlip DE, Jamerson K, Henrich W, Reid DM, et al. Stenting and medical therapy for atherosclerotic renal-artery stenosis. $N$ Engl J Med. (2014) 370:13-22. doi: 10.1056/NEJMoa1310753

7. Raman G, Adam GP, Halladay CW, Langberg VN, Azodo IA, Balk EM. Comparative effectiveness of management strategies for renal artery stenosis: an updated systematic review. Ann Intern Med. (2016) 165:635-49. doi: 10. 7326/m16-1053

8. de Leeuw PW, Postma CT, Spiering W, Kroon AA. Atherosclerotic renal artery stenosis: should we intervene earlier? Curr Hypertens Rep. (2018) 20:35. doi: 10.1007/s11906-018-0829-3

9. Prince M, Tafur JD, White CJ. When and how should we revascularize patients with atherosclerotic renal artery stenosis? JACC Cardiovasc Interv. (2019) 12:505-17. doi: 10.1016/j.jcin.2018.10.023

10. Tafur JD, White CJ. Renal artery stenosis: when to revascularize in 2017. Curr Probl Cardiol. (2017) 42:110-35. doi: 10.1016/j.cpcardiol.2017.01.004

11. Textor SC, Glockner JF, Lerman LO, Misra S, McKusick MA, Riederer SJ, et al. The use of magnetic resonance to evaluate tissue oxygenation in renal artery stenosis. J Am Soc Nephrol. (2008) 19:780-8. doi: 10.1681/asn.2007040420

12. Saad A, Herrmann SM, Crane J, Glockner JF, McKusick MA, Misra S, et al. Stent revascularization restores cortical blood flow and reverses tissue hypoxia in atherosclerotic renal artery stenosis but fails to reverse inflammatory

\section{AUTHOR CONTRIBUTIONS}

ZL, BZ, and LL contributed in implementing and writing. RW, $\mathrm{GN}$, and $\mathrm{ZY}$ contributed in implementing and data analysis. YZ, XT, JQ, and MY contributed in supervising. All authors contributed to the article and approved the submitted version.

\section{FUNDING}

This work was supported by National Key R\&D Program of China (grant no. 2017YFC0109105), the Scientific Research Seed Fund of Peking University First Hospital (grant no. 2018SF023), Youth clinical research project of Peking University First Hospital (grant no. 2018CR16), and Interdisciplinary clinical research project of Peking University First Hospital (grant no. 2018CR33).

pathways or glomerular filtration rate. Circ Cardiovasc Interv. (2013) 6:428-35. doi: 10.1161/circinterventions.113.000219

13. Manohar S, Hamadah A, Herrmann SM, Textor SC. Total renal artery occlusion: recovery of function after revascularization. Am J Kidney Dis. (2018) 71:748-53. doi: 10.1053/j.ajkd.2017.11.014

14. Gloviczki ML, Saad A, Textor SC. Blood oxygen level-dependent (BOLD) MRI analysis in atherosclerotic renal artery stenosis. Curr Opin Nephrol Hypertens. (2013) 22:519-24. doi: 10.1097/MNH.0b013e32836 $400 \mathrm{~b} 2$

15. Batide-Alanore A, Azizi M, Froissart M, Raynaud A, Plouin PF. Split renal function outcome after renal angioplasty in patients with unilateral renal artery stenosis. J Am Soc Nephrol. (2001) 12:1235-41. doi: 10.1681/asn. v1261235

16. Saeed A, Fortuna EN, Jensen G. Split renal function in patients with unilateral atherosclerotic renal artery stenosis-effect of renal angioplasty. Clin Kidney J. (2017) 10:496-502. doi: 10.1093/ckj/sfx052

17. Leesar MA, Varma J, Shapira A, Fahsah I, Raza ST, Elghoul Z, et al. Prediction of hypertension improvement after stenting of renal artery stenosis: comparative accuracy of translesional pressure gradients, intravascular ultrasound, and angiography. J Am Coll Cardiol. (2009) 53:2363-71. doi: 10.1016/j.jacc.2009.03.031

Conflict of Interest: The authors declare that the research was conducted in the absence of any commercial or financial relationships that could be construed as a potential conflict of interest.

Publisher's Note: All claims expressed in this article are solely those of the authors and do not necessarily represent those of their affiliated organizations, or those of the publisher, the editors and the reviewers. Any product that may be evaluated in this article, or claim that may be made by its manufacturer, is not guaranteed or endorsed by the publisher.

Copyright (c) 2022 Lin, Zhang, Lin, Wang, Niu, Yan, Zou, Tong, Qiu and Yang. This is an open-access article distributed under the terms of the Creative Commons Attribution License (CC BY). The use, distribution or reproduction in other forums is permitted, provided the original author(s) and the copyright owner(s) are credited and that the original publication in this journal is cited, in accordance with accepted academic practice. No use, distribution or reproduction is permitted which does not comply with these terms. 